\title{
ATTENTIONAL TRACKING OF MULTIPLE VEHICLES IN A HIGHWAY DRIVING SCENARIO
}

\author{
Martin Lochner \& Lana Trick \\ Department of Psychology, University of Guelph \\ Guelph, Ontario, Canada \\ E-mail: mlochner@uoguelph.ca
}

\begin{abstract}
Summary: In this paper we introduce a 'vehicle tracking' task, which tests the ability of a driver to track the location of multiple vehicles on the roadway. Based on the 'multiple object tracking' task (Pylyshyn \& Storm, 1988), the vehicle tracking task presents the driver with an array of identical vehicles immediately in front of the subject vehicle. The task consists of three distinct stages: encoding, during which the target vehicles are indicated to the driver; tracking, during which all vehicles change lanes in a random order; and report, during which the participant indicates the final location of the target vehicles. Using this methodology, we test the accuracy with which university-aged drivers can track multiple vehicles in a 3-lane highway driving scenario. Our particular interest in this paper is how the ability to attend to multiple vehicles changes as task load increases.
\end{abstract}

\section{OBJECTIVES}

Driving an automobile is a classic example of how visual attention needs to be distributed to multiple foci, in order to facilitate safe performance of a complex task. On a typical day, a driver will encounter many situations in which simultaneous, or near simultaneous attention to multiple moving vehicles or people is necessary in order to avoid a collision; for example, when performing a passing maneuver on a busy highway, or parking in a crowded lot. Consider the common occurrence of an 'unprotected' left turn, at which a driver must turn across rapidly moving traffic. The driver must attend to the oncoming vehicles, possibly in multiple lanes; there is also the possibility of pedestrian traffic, bicycles, and vehicles encroaching from the rear, which should be monitored in the mirrors. This, added with constant vigilance for unexpected events, sums to create a situation in which the driver must rapidly develop and maintain a mental model of the relevant entities in the driving situation.

The ability to assess the number of physical locations that an adult driver can attend to, with a reliable understanding of how this is affected by situational factors such as driver experience and task load, would broaden our understanding of the risks present in a given driving scenario. In this paper we introduce a task that provides one possible way to measure this ability, discuss some possible uses of the task to the driving research community, and describe our planned future directions for research into the ability to attend to multiple entities in the driving environment. The specific objectives of this study are to determine first, the number of vehicles that a driver can track (where 'track' means to keep track of the physical location of the vehicle), and second, how the typical requirements placed upon the driver impact the ability to track multiple vehicles. 


\section{Multiple Vehicle Tracking}

The vehicle tracking task presented here is a result of our efforts to re-create the classical 'multiple object tracking' task in a vehicular environment, and is part of an ongoing attempt to demonstrate how much information is available to the driver, regarding the foci of distributed attention in a visual scene. The multiple object tracking task (Pylyshyn \& Storm, 1988) is a basic paradigm that requires the observer to track the locations of multiple target circles as they move about a monochrome computer display. Healthy adults can generally track between 4 and 5 items amongst an equal number of non-target items (Pylyshyn, 2009), though this number is reduced somewhat in elderly populations (Trick et al., 2005; 2009). As Trick et al. (2005) point out, reduced object tracking ability in elderly populations may be closely related to increased collision risk at intersections (e.g. McGwin \& Brown, 1999) due to a reduced ability to develop and maintain an accurate mental representation of the vehicles' locations in physical space. Though the ability to track basic objects in a simple display has been well studied, research into how objects are tracked in more complex environments is relatively scarce, and has, to date, not been investigated in the driving environment, despite the fact that several authors write that the driving environment is exactly the type of situation where object tracking is most relevant.

In this first experiment, we are interested in demonstrating the number of vehicles that can be tracked in a typical highway driving scenario, and further, how this performance is affected by the requirement of steering and maintaining a specific headway distance. Considering that the vehicle tracking task described here is markedly more complex than the original generic object tracking paradigm, it is expected that tracking accuracy will fall somewhere below the $\sim 90 \%$ accuracy that is found in a standard desktop-computing environment (e.g. Pylyshyn \& Storm, 1988; Yantis, 1993). Further, given that steering and maintaining the speed of a vehicle are considered 'ambient' attentional tasks (Wickens, 2002), and that the addition of a secondary task has previously been shown to reduce generic object tracking performance (e.g. Tombu \& Seiffert, 2008), it is expected that the inclusion of steering and headway requirements will result in a significant reduction in the number of vehicles that can be tracked.

\section{METHOD}

\section{Subjects}

Twenty-nine undergraduate students between the ages of $18-25$ from the University of Guelph participant pool participated in this study. Participants had normal or corrected to normal vision, and received 1 course credit for their participation. All participants were screened for medical issues that could be dangerous in a driving simulator (epilepsy, heart conditions), and had at least 2 years of licensed driving experience.

\section{Design}

This study employed a $3 \times 3$ repeated measures design. The first independent variable was the number of targets (1,3 or 4), and the second independent variable was task load. The three levels of task load were: 'object tracking only' (no steering or headway maintenance required), 'multiple-task' (steering and headway maintenance required, in addition to object tracking), and 
'baseline: driving only' (steering and headway maintenance required, with no object tracking task). The experiment consisted of 3 blocks of 30 trials each, where the order of blocks is counterbalanced. Accuracy at the tracking task was measured using a full-report methodology, where the participant attempted to locate all of the cued targets. The main dependent variable was the percentage of target vehicles that were correctly identified (e.g. $3 / 4$ targets $=75 \%$ accuracy). Additionally, steering performance and speed maintenance were measured.

\section{Apparatus and Stimuli}

Tracking tasks took take place within a Drivesafety DS 600C fixed-base driving simulator. The simulator body is a modified Saturn sedan, and the projection system provides a 300 -degree wrap-around display consisting of 5 screens in the front and 1 in the back (250 and 50 degrees, respectively). The simulator display operates at 256 colors, with a refresh rate of $60 \mathrm{hz}$. A standard 3-lane "freeway" style roadway-simulation, with no turns, was modified such that 9 identical blue cars appear in front of the participant vehicle in a 3 x 3 grid, as shown in Figure 1. In the 'object tracking only' condition, vehicles in the row closest to the driver (row 1) subtended an average visual angle of $2.97^{\circ}$, ranging from $2.01^{\circ}$ at the most distant position, to $3.44^{\circ}$ at the closest position. The vehicles in row 2 and 3 subtended an average of $2.1^{\circ}$ degrees (range $1.9^{\circ}$ to $2.3^{\circ}$ ) and $1.86^{\circ}$ (range $1.72^{\circ}$ to $2^{\circ}$ ), respectively. In the 'multiple task' condition the precise visual angle of the object vehicles was similar to that in the 'object tracking only' condition, but depended on participants' performance on headway maintenance task.
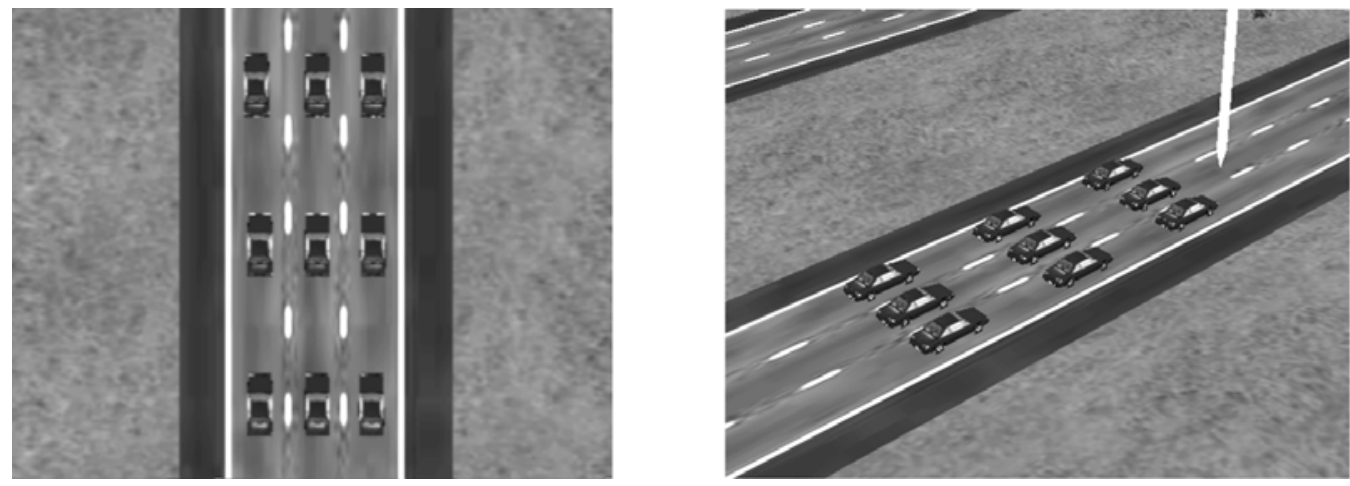

Figure 1. Vehicle tracking stimuli from 'birds eye' (left) and isometric (right) view angles

\section{Procedure}

Upon arrival, participants were given the information/consent forms, as well as a Simulator Sickness Questionnaire (Kennedy et al., 1993) in order to determine their susceptibility to simulator sickness (i.e. motion sickness due to simulator usage). Participants with a high likelihood of becoming ill during the simulation did not take part in the study, but received full credit nonetheless. Once the participant was seated, the experiment began with the simulator in a stopped position, with the 9 object vehicles directly ahead. The subject vehicle was placed between the left and middle highway lanes, directly on top of the dotted line, in order to maximize visibility for all 9 object vehicles. The participant was initially given a 5-minute training period to become familiar with the simulator and task. In the 'object tracking only' condition the subject vehicle, as well as the 9 object vehicles, accelerated automatically to 60 
$\mathrm{km} / \mathrm{h}$. In the 'multi-task' condition the 9 object vehicles accelerated automatically to $60 \mathrm{~km} / \mathrm{h}$, and the participant was required to maintain headway and lane position behind the object vehicles. At the beginning of each trial the target vehicles (1, 3 or 4) flashed off and on five times, indicating which vehicles were to be tracked. At this point the vehicles began changing lane-position laterally on the roadway at a rate of 1 change every 2 seconds. Vehicles were allowed to change positions without maneuvering to avoid one-another. The lane-changing continued for 45 seconds, during which time the vehicles progressed through a random ordering of all possible lane positions. When the lane changes were complete, the simulation was paused, and the participant was asked to indicate the final location of the target vehicles, using a ledger designed for this purpose. When the trial was finished, the next trial was started via a button press, and the experiment continued until completion. Each trial took approximately one minute, including 5 seconds to cue the target vehicles, 45 seconds of tracking, $\sim 5$ seconds to report, and 5 seconds to reset the vehicle positions for the next trial.

\section{RESULTS}

No data in this study were lost as a result of simulator sickness.

\section{Accuracy Data}

A $3 \times 2$ (number of targets $x$ task load) ANOVA was performed on the object tracking accuracy data (no accuracy data was collected for the Baseline Drive: No Tracking condition). Score sheets for 6 participants were misplaced and are therefore left out of the analysis. The main effect of number of targets was significant at $F(2,44)=28.676, \mathrm{p}<.000$, partial $\eta^{2}=.566$, indicating that tracking accuracy was significantly lower when there were more targets to track. The main effect of task load was also significant at $F(1,22)=37.543, p<.000$, partial $\eta^{2}=.631$, indicating that accuracy was lower when the subject was required to steer and maintain the simulator vehicle's speed. Finally there was a task load x number of targets interaction, $F(2$, $44)=3.781, p<.05$, partial $\eta^{2}=.147$. When subjects were required to steer and maintain the vehicle's speed, accuracy at tracking 3 or 4 vehicles was lower than when the subject was not required to steer and maintain speed: this is illustrated in Figure 2.

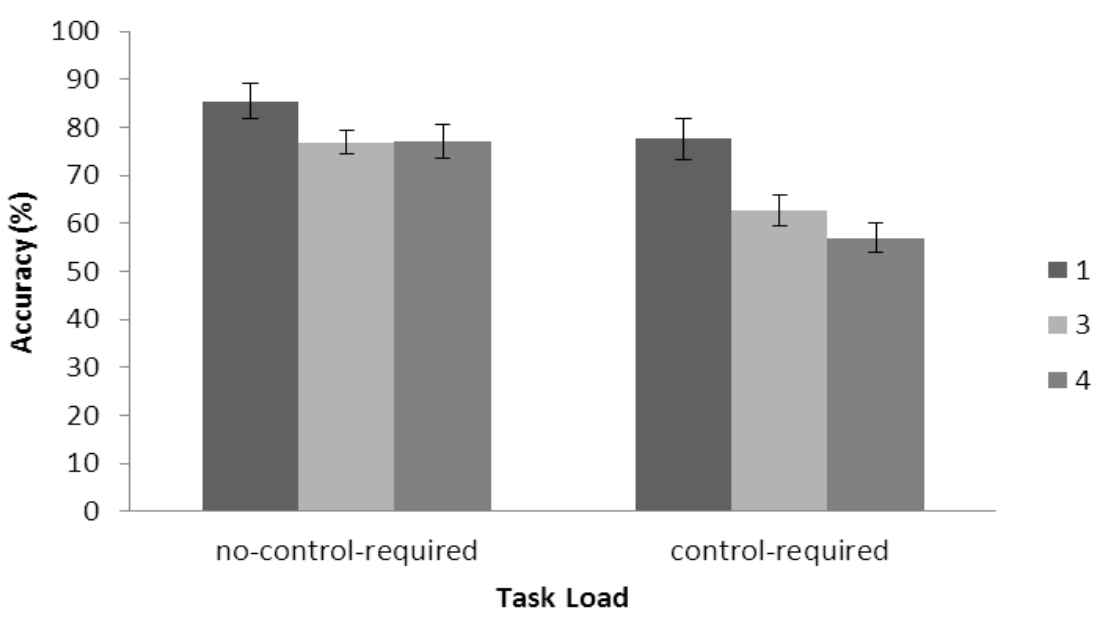

Figure 2. Accuracy Data (Number of Targets X Task Load)

(Error bars represent standard error of the mean) 


\section{Lane Position and Headway Data}

Lane position and headway data from all 29 participants were analyzed. The data for standard deviation of lane position (SDLP) and standard deviation of headway distance (both measured in square meters) were subjected to an outlier analysis, and all scores greater than 2.5 standard deviations from the mean were removed from the analysis. This resulted in the removal of a total of 3 data points. Two simple ANOVAs were performed, one for the lane deviations, and one for the headway data. Deviations in lane position were significantly affected by the number of targets manipulation, $F(2,54)=4.851, p<.05$, partial $\eta^{2}=.152$. Likewise, drivers' ability to maintain a consistent headway (standard deviation of headway distance) was significantly influenced by the number of targets to be tracked, $F(2,54)=3.838, p<.05$, partial $\eta^{2}=.124$. Baseline data for driving with no task are also provided, but were not included in the ANOVAs.
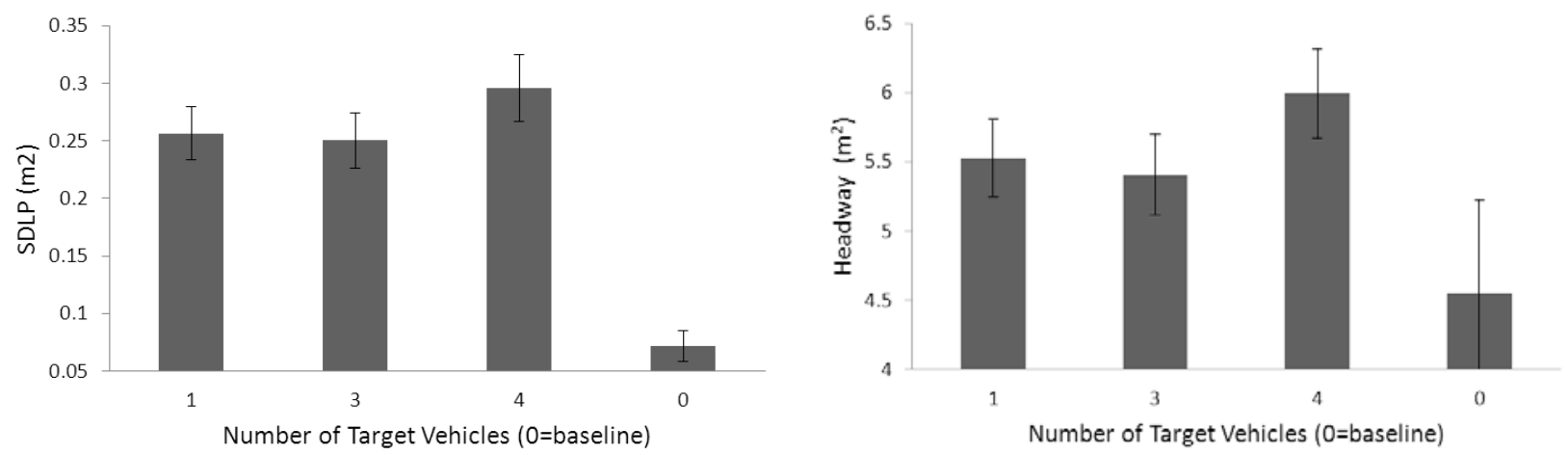

Figure 3. Mean standard deviation of lane position (left) and headway distance (right) (Error bars represent standard error of the mean)

These results indicate a number of interesting findings. Accuracy at tracking is generally very high when no vehicle control (steering, speed maintenance) is required ( $85 \%$ for 1 target, and $\sim 80 \%$ for 3 and 4 targets). This is only somewhat lower than what is found in classical multiple object tracking studies (e.g. Pylyshyn \& Storm, 1988), indicating that tracking the location of multiple vehicles is, in itself, not much more difficult than tracking generic objects. Additionally, there was a significant reduction in tracking accuracy at 3 and 4 targets (but not at 1 target) when vehicle control requirements are introduced. Because steering and maintaining vehicle speed are considered to be 'ambient' attentional tasks (Wickens 2002), and because they interfere with tracking performance, it may be concluded that tracking is an ambient-attention task, or at least contains a substantial ambient-attentional component. Finally, it is also evident that the requirement of tracking multiple vehicles is detrimental our ability to steer the vehicle and maintain a desired headway. This highlights the danger of assuming that individuals can track " 4 or 5 " independent targets when operating a motor vehicle.

\section{CONCLUSIONS}

In this paper we have presented a novel task that enables us to measure the number of discrete, independent vehicle locations that can be tracked in a typical highway driving scenario. Further, we investigated how the ability to track multiple vehicles is impacted by the standard requirements of steering a vehicle and maintaining a constant headway. The current experiment 
presents the general paradigm, and provides a foundation for further investigation into how much, and what type of information is available to drivers, regarding moving entities in the driving environment. This is useful because of the complex nature of driving an automobile in traffic, and the fact that understanding the limitations on drivers' ability to attend to multiple locations will potentially allow for greater automobile safety. Given that the task is sensitive to attentional factors such as task load, vehicle tracking ability could potentially be used as a metric for assessing the cognitive demand of a given in-vehicle task or device, or other activity. Additionally, the task presented here has an advantage over competing measures because it is a dynamic task, requiring prolonged, continuous attention to the target items, rather than a brief, momentary application of attention.

Our research is currently geared towards learning how much, and what kind of information is available regarding the tracked vehicles. In particular, we are interested in testing drivers' ability to detect subtle changes in the tracking set for target and non-target vehicles, as well as drivers' ability to respond to sudden brake onsets for target vs. non-target vehicles. Both of these investigations could potentially improve safety in the driving environment, by developing a better understanding of how information for situation-relevant vehicles is processed. Further, we are interested in how heterogeneity in the vehicle-tracking set will affect tracking performance. In a typical roadway environment, the driver is faced with a heterogeneous array of visual stimuli, rather than the identical items used in the current experiment. There is evidence that unique color information can benefit object tracking performance (e.g. Makovski et al., 2009), though this advantage disappears when a secondary executive task is introduced. In another upcoming study we will investigate whether this advantage for unique objects exists in a driving environment, and further, if it persists when vehicle-control requirements are introduced.

Finally, it will be interesting to model increasingly realistic driving scenarios, such as the left turn example given at the beginning of this paper, or other complex highway scenarios such as passing or crash avoidance. Given that these scenarios involve attending to multiple physical locations, and that this ability is influenced by factors such as task load, or age and experience, the vehicle tracking task introduced here is potentially a useful metric for the investigation of multiple attentional foci in complex driving scenarios.

\section{ACKNOWLEDGEMENTS}

We would like to thank the members of the Driving Research In Virtual Environments lab at the University of Guelph for their help with this study, as well as the following agencies, which have provided funding grants to the second author on this paper: the Canadian Foundation for Innovation, Ontario Innovation Trust, Auto21: Network Centres of Excellence, and the Natural Sciences and Engineering Research Council of Canada.

\section{REFERENCES}

Awh, E., \& Pashler, H. (2000). Evidence for split attentional foci. Journal of Experimental Psychology: Human Perception and Performance. Vol 26(2), pp. 834-846.

Kennedy, R.S., Lane, N.E. (1993). Simulator Sickness Questionnaire: An Enhanced Method for Quantifying Simultator Sickness. The International Journal of Aviation Psychology, 3(3), 
203-220.

Makovski, Tal; Jiang, Yuhong V. (2009). Feature binding in attentive tracking of distinct objects. Visual Cognition. Vol 17(1-2), p. 180-194.

Michon, J. A. (1985). A critical view of driver behaviour models: What do we know, what should we do? Human behaviour and traffic safety, 485 - 520, New York: Plenum Press.

McGwin, G. \& Brown, D. (1999). Characteristics of traffic crashes among young, middle-aged, and older drivers. Accident Analysis and Prevention, 31, 181-189.

Pylyshyn, Z. \& Storm, R. (1988). Tracking multiple independent targets: Evidence for a parallel tracking mechanism. Spatial Vision, 3 (3), 179-197.

Pylyshyn, Z. (2004). Some puzzling findings in multiple object tracking: I. Tracking without keeping track of object identities. Visual Cognition, 11 (7), 801-822.

Pylyshyn, Z. (2009). Perception, Representation, and the World: The FINST That Binds. In D. Dedrick \& L. Trick (Eds.), Computation, Cognition, and Pylyshyn (pp. 3-48). Cambridge, MA: MIT Press.

Scholl, Brian J. (2009). What have we learned about attention from multiple-object tracking (and vice versa)? in Dedrick, Don; Trick, Lana (eds) Computation, cognition, and Pylyshyn. (pp. 49-77). Cambridge, MA, US: MIT Press. xvii, 344 pp.

Tombu, M., \& Seiffert, A. (2008). Attentional costs in multiple-object tracking. Cognition, Vol 108(1), pp. 1-25.

Trick, L.M., Perl, T., Sethi, N. (2005). Age related differences in multiple object tracking. The Journals of Gerontology: Series B: Psychological Sciences and Social Sciences. Vol. 60B(2), pp. 102-105.

Trick, L.M., Hollinsworth, H., \& Brodeur, D. (2009). Multiple-object tracking across the lifespan: Do different factors contribute to diminished performance in different age groups? In Don Dedrick and Lana Trick (Eds). Computation, Cognition, and Pylyshyn. (pp. 79-99). MIT press.

Wickens, Christopher D. (2002). Multiple resources and performance prediction. Theoretical Issues in Ergonomics Science. Vol 3(2), pp.159-177.

Yantis, Steven. (1993). Stimulus-driven attentional capture and attentional control settings. Journal of Experimental Psychology: Human Perception and Performance. Vol 19(3), pp. 676-681. 